\title{
On the Construction of Solvable Models of the Many Body Problem
}

\author{
D. SCHÜTTE \\ Institut für Theoretische Kernphysik der Universität Bonn, Germany \\ (Z. Naturforsch. 28 a, 396-403 [1973] ; received 26 January 1973) \\ Dedicated to K. BLEULER on his 60th birthday
}

\begin{abstract}
The construction of subalgebras of the set of bilinear products of fermion operators which allow the formation of physically relevant solvable models is investigated. The subalgebras are assumed to be semisimple, hermitean closed and compatible with angular momentum. One obtains two classes of subalgebras, i) the wellknown set of spherical tensors of rank zero and ii) a new class of subalgebras which contain the angular momentum operators. The construction procedure for subalgebras of the second kind is presented in detail (hereby, one starts with the abstract type of the subalgebra and defines the physical model space by the carrier space of a representation) and a number of examples, which provide models not known from the literature, is given.
\end{abstract}

\section{Introduction}

The investigation of solvable models is a useful tool for studying the structure of the solutions of the many body problem and to test the validity of different many body approximation techniques. Examples of models suitable for such applications are the pairing Hamiltonian ${ }^{1}$, Elliott's su(3) model of light nuclei containing a quadrupole force ${ }^{2,3}$, Lipkins two shell model with monopole forces ${ }^{4}$ or pairing plus monopole forces ${ }^{5}$ and Agassi's four shell model with monopole forces for protons and neutrons ${ }^{6}$.

All these models have their basis in the existence of suitable subalgebras of the Lie algebra $L_{N}$ generated by the bilinear products of creation and annihilation operators [of the type so $(2 N) *$ if a finite number $N$ of such operators is considered]. This allows to obtain rigorous solutions by group theoretical methods.

In fact, most of the group theoretical techniques of the many body problem are based on such subalgebras. Consequently, the problem of constructing physically relevant subalgebras of $L_{\mathrm{N}}$ has been discussed extensively in the literature in connection with applications in atomic and nuclear physics. Besides to the more schematic examples quoted in the beginning we refer to the following works. For pure configurations (i.e. for a Fock space based on a single shell) such group theoretical problems have been discussed by RACAH ${ }^{8}$, FLOWERS ${ }^{9}$ and JUdD ${ }^{10}$, yielding a general survey on all physically relevant

* We use the notation of Helgason ${ }^{7}$ for characterizing Lie algebras. subalgebras of $L_{N}$. For the case of mixed configurations (different shells) we mention, besides the su(3) model of Elliott ${ }^{2}$, applications to two shells by Wybourne ${ }^{11}$ and Feneuille ${ }^{12}$ and the more general investigations of MOSHINSKY ${ }^{13}$ and KRAMER and Seligman ${ }^{14}$. More details of the extended literature in this connection can be found in the reviews of JUdD ${ }^{15}$, WybOURne ${ }^{16}$ and Kramer and MOSHINSKY ${ }^{17}$. (For more general applications of Lie algebraic methods we refer to the standard literature ${ }^{18-21}$.)

Characteristic for all these applications is that the physically relevant subalgebras of $L_{X}$ are constructed by starting from a given configuration (i.e. a Fock space defined by given shell combinations) and by working out suitable chains of subalgebras of so $(2 N)$ [or $u(N)]$ ending with the angular momentum algebra so(3).The elements of each of these subalgebras may be used to define model interactions (which may be relevant parts of the realistic interactions) and to define classifications of the many body states. For the case of mixed configurations it is, however, difficult to obtain in this way a systematic survey on all subalgebras suitable for the construction of physically relevant models.

It is the main purpose of this paper to show that such a survey can be provided by inverting the described construction procedure. This yields an infinite number of different models and one has to decide from case to case, whether the construction has physical meaning in terms of mixed configurations.

In order to make clear this construction, we first analyse the mathematical properties of physically relevant subalgebras (Section II). We derive how 
by natural, physically motivated restrictions (semiimplicity, compactness, compatibility with angular momentum) one is lead to two types of subalgebras, namely either to the set of all bilinear products which commute with the angular momentum or to such subalgebras which contain the angular momentum operators. For the construction of subalgebras of the first type one may proceed in the usual way by starting from a given configuration. Section III gives a brief survey of the structure of such subalgebras yielding in general wellknown models.

In Section IV we describe the construction of subalgebras of the second type. Herefore, we start with the abstract type of the subalgebra, we define the Fock space by the carrier space of a representation (which can be chosen), and we specify the angular momentum structure of that Fock space by a special subalgebra [of the type so(3)] of the abstract Lie algebra (which again can be given arbitrarily).

In Section V some details of the structure of these subalgebras are analysed. We present a number of examples of subalgebras of the second type in terms of their abstract characteristics.

Our construction will yield as special cases the known subalgebras of $L_{N}$ (e. g. those for a pure configuration and the Elliott model). However, for the case of mixed configurations one is lead in general to the definition of subalgebras and models which have not been discussed in the literature. E. g., we are able to define a new su(4) model for the configuration $1 / 2,3 / 2,5 / 2,7 / 2$.

In this paper, we only give an abstract classification of the models, which still have to be treated in detail by special group theoretical methods in order to derive the concrete properties of the models. Such a more concrete analysis - for the su(4) example will be the subject of a future publication.

\section{Definition and Structure of Physically Relevant Subalgebras}

Consider a Fock space generated by $N$ creation operators $a_{\alpha}{ }^{+}(\alpha=1, \ldots, N)$ from the vacuum and call $L_{N} \cong$ so $(2 N)$ the Lie algebra generated by the set of bilinear products of creation and annihilation operators, i. e.

$L_{N}=\left(a_{\alpha}^{+} a_{\beta}-a_{\beta} a_{\alpha}^{+}, a_{\alpha}^{+} a_{\beta}^{+}, a_{\alpha} a_{\beta},(\alpha, \beta=1, \ldots, N)\right)$.
Our aim is to construct subalgebras $L$ of $L_{N}$ (i.e. subspaces of $L_{N}$ which are closed with respect to the formation of commutators) which are, on the one hand, simple enough to be treatable by standard group theoretical methods and, on the other hand, allow the construction of physically relevant operators (model hamiltonians) with the help of suitable polynomials in elements of $L$.

The first condition will be fulfilled if $L$ is semisimple. The theory of such Lie algebras, especially that of their representations is well developped (see e. g. Refs. ${ }^{7,19,20}$ ). Also we will assume that $L$ is hermitean closed, i.e. $L^{+}=L$. Physically, this guarantees the possibility to construct hermitean operators. Mathematically, this assumption has the consequence that $L$ constitutes a representation of an (abstract) semisimple Lie algebra in its compact real form which is unitary with respect to the physically given scalar product of the Fock space.

In the notation of HeLGASON ${ }^{7}$, this abstract type of $L$ is given by so $(n), \operatorname{su}(n)$ or $\operatorname{sp}(n)$ (or the exceptional algebras, see Cartan's list $\left.{ }^{7}\right)$ and by direct sums of such Lie algebras.

A main restriction on $L$ will result from the necessity of physical relevance. A basical condition any model has to fulfil is rotational invariance. Therefore, we will postulate that $L$ should allow the generation of operators which commute with angular momentum. In order to guarantee this we must first specify the angular momentum structure of $L_{N}$.

Let us assume that the single particle space is closed with respect to angular momentum, i. e. the quantum number $\alpha$ can be specified as

$\alpha=k j m$

$\left(m=-j, \ldots, j, k=1, \ldots, n_{j}, j=j_{1}, \ldots, j_{S}\right)$.

It is wellkown that $L_{N}$ can then be decomposed into spherical tensors (tensor operators) ${ }^{21}$

$$
\begin{aligned}
L_{N}=\left(A_{\mu}^{J M}, M\right. & =-J, \ldots, J, \\
\mu & \left.=1, \ldots, \mu_{J}, J=J_{1}, \ldots, J_{A}\right)
\end{aligned}
$$

where the operator $A_{\mu}^{J M}$ are, in a suitable enumeration, the coupled operators ${ }^{15,21-23}$

$$
\left(\boldsymbol{a}_{k j}^{+} \quad a_{k^{\prime} j^{\prime}}-\boldsymbol{a}_{k^{\prime} j^{\prime}} \boldsymbol{a}_{k j}^{-}\right)^{J M},\left(a_{k j}^{+} a_{k^{\prime} j^{\prime}}^{+}\right)^{J M},\left(a_{k j}, a_{k^{\prime} j^{\prime}}\right)^{J M} .
$$

For any infinitesimal rotation $e$ [i. e. e $\in$ so $(3)]$ and its representation $d(e)$ on the Fock space we have then that

$$
\left[d(e), A_{\mu}^{J M}\right]=\sum_{M^{\prime}} d_{M M^{\prime}}^{J}(e) A_{\mu}^{J M^{\prime}} .
$$


(We denote by $d^{J}$ the standard irreducible representation of so (3) on $C^{2 J+1}$.)

We now impose in the following the condition on $L$ that also this subalgebra of $L_{N}$ is completely decomposable into spherical tensors, i. e. there exist $B_{v}^{J M}$ such that

$$
\begin{aligned}
L=\left(B_{v}^{J M}, M=-J, \ldots, J,\right. & \\
& \left.v=1, \ldots, v_{J}, \quad J=J_{1}, \ldots, J_{B}\right)
\end{aligned}
$$

where the operators $B_{v}^{J M}$ are certain linear combinations of the operators $A_{\mu}^{J M}$ with $J$, M fixed.

This guarantees that, up to second order, the following operators are rotational invariant, i. e. commute with all $d(e)[e \in \mathrm{so}(3)]$ :

$$
\begin{array}{r}
B_{v}^{00}\left(v=1, \ldots, v_{0}\right), \sum_{M}(-1)^{M} B_{v}^{J M} B_{v^{\prime}}^{J-M} \\
\left(v, v^{\prime}=1, \ldots, v_{J}, J=J_{1}, \ldots, J_{B}\right) .
\end{array}
$$

The above assumption ( $L$ semisimple, conditions (5), i. e. $\left.[d(e), L] C L, L^{+}=L\right)$ now lead, by the application of standard techniques of the theory of Lie algebras, to the following structure of $L$ :

There are two alternatives

i) $L$ commutes with all $d(e)[e \in \mathrm{so}(3)]$.

ii) All $d(e)$ are contained in $L$, if the Fock space is suitably restricted, i. e. if one takes into account for the definition of $L_{N}$ a suitable subset of the creation and annihilation operators such that the elements of $L$ remain unaltered.

The proof, which is provided by a generalization of the wellknown theorem, that any derivation of a semisimple Lie algebra is inner ${ }^{7,20}$, will not be given here ${ }^{25}$. It is not necessary for our further construction of physically relevant subalgebras of $L_{N}$, it is only interesting in order to judge the generality of these constructions. Given the two alternatives i) and ii), a systematic construction of corresponding subalgebras $L$ is now possible. Hereby, for the first case, $L_{N}$ can be given, whereas in case ii) the type of shell combinations [Eq. (2)] has to chosen suitably, and it has to be checked later whether physically interesting systems can be described in the corresponding Fock spaces.

\section{The Centralizer of the Angular Momentum}

We first discuss briefly the subalgebras of type i).

From $[d(e), L]=0[e \in$ so $(3)]$ we see that the maximum subalgebra of this type is given by the centralizer $z_{\mathrm{a}}$ of the angular momentum, i. e. by the set of all elements of $L_{N}$ which commute with all $d(e)[e \varepsilon$ so (3) $]$. Any other $L$ obeying i) is then a subalgebra of $z_{\mathrm{a}}$. Equation (4) tells us that $z_{\mathrm{a}}$ consists of all spherical tensors of rank zero, i. e.

$$
z_{\mathrm{a}}=\left(A_{\mu}^{00}, \mu=1, \ldots, \mu_{0}\right) .
$$

Taking into account the $k j m$ classification of the single particle states, we obtain for $z_{\mathrm{a}}$ the direct sum decomposition

$$
z_{\mathrm{a}}=\bigoplus_{j=j_{1}}^{j_{\mathrm{S}}} z_{j}
$$

with

$$
\begin{gathered}
z_{j}=\sum_{m}\left(a_{k j m}^{+} a_{k^{\prime} j m}-a_{k^{\prime} j m} \boldsymbol{a}_{k j m}^{+}\right), \sum_{m} s_{m} \boldsymbol{a}_{k j m}^{+} a_{k^{\prime} j-m}^{+}, \\
\left.\sum_{m} s_{m} a_{k j m} a_{k^{\prime} j-m, k}, k^{\prime}=1, \ldots, n_{j}\right), \\
{\left[s_{m}=(-1)^{j-m}\right] .}
\end{gathered}
$$

It may be checked easily (see Refs. ${ }^{21,24,25}$ ) that $z_{\text {j }}$ has the structure

$$
z_{j} \cong \begin{aligned}
& \operatorname{sp}\left(n_{j}\right) \quad \text { for halfinteger } j, \\
& \mathrm{so}\left(2 n_{j}\right) \text { for integer } j .
\end{aligned}
$$

Hereby, halfinteger $j$ means that $j$ and $d(\mathrm{e})$ correspond to the total angular momentum, integer $j$ means that we are treating $j$ and $d(e)$ as orbital angular momentum. Restricting $z_{j}$ to elements which commute with the particle number operator $N_{j}^{o p}=\sum_{k, m} a_{k j m}^{+} a_{k j m}$ and taking out $N_{j}^{\text {op }}$ itself yields a subalgebra

$$
\begin{aligned}
\boldsymbol{z}_{j}^{0} & =\left(\sum_{m} a_{k j m}^{+} a_{k^{\prime} j m}-\delta_{k k^{\prime}} / n_{j} N_{j}^{\text {op }}, k, k^{\prime}=1, \ldots n_{j}\right) \\
& \cong \operatorname{su}\left(n_{j}\right)
\end{aligned}
$$

$z_{j}$ and $z_{j}^{0}$ obviously are subalgebras of $L_{N}$ which fall into the category i). They are wellknown from the literature (see e.g. ${ }^{21}$ ) and we only mention schematically some applications based on $z_{\mathrm{a}}$.

In the case of halfinteger $j$ we have the pairing models for n shells, which assume $n_{j}=1, S=n$, the operators (6) giving the wellknown pairing interaction ${ }^{1,21}$. The monopole models for two shells ${ }^{4}$ and four shells ${ }^{6}$ exploit the possibilities $S=1$, $n_{j}=2$ and 4 by considering operators out of $z_{j}{ }^{0}$.

The pairing plus monopole model $^{5}$ is constructed by taking elements of $z_{j}$ for $n_{j}=2, S=1$. The operators of $z_{j}$ are also used for the classification of states. Thus, $z_{j}$ for $n_{j}=S=1$ defines seniority ${ }^{21}, z_{j}{ }^{0}$ for $n_{j}=1, S=2$ may be interpreted as isospin ${ }^{21}$, $z_{j}$ being a generalization of both concepts in this case ${ }^{26}$. 
For integer $j$ (orbital angular momentum interpretation of $d$ ) and $n_{j}=4, S=1, z_{j}{ }^{0}$ yields the su (4) classification scheme for light nuclei (Wigner supermultiplet ${ }^{27}$ ) which may be generalized to $z_{j} \cong$ so (8) (see Ref. ${ }^{28}$ ).

For the atomic nucleus, one should mention the application of $z_{\mathrm{j}}{ }^{0}$ and $z_{j}$ in the case of $n_{j}=2, S=1$, as described e. g. in Ref. ${ }^{15}$.

\section{Subalgebras Containing the Angular Momentum}

We shall now describe the construction of subalgebras of $L_{N}$ which are of the type ii). For simplicity, we will assume that $L$ is build up only of elements which do not change the particle number, i.e. we will assume $L$ to be subalgebra of $L_{N}{ }^{0}$, where

$L_{N}^{0}=\left(a_{\alpha}^{+} a_{\beta}-a_{\beta} a_{\alpha}^{+}, \alpha, \beta=1, \ldots, N\right) \cong u(N)$.

More general subalgebras may be easily obtained, however, it has been not possible to find physically interesting examples.

Also, we will make use of the fact that the structure $L_{N}{ }^{0} \cong u(N)$ results from the (Lie algebra) isomorphism $\sigma$ between $N \times N$ matrices $A_{\alpha \beta}$ and $L_{N}{ }^{0}$ given by (we use summing convention over doubly appearing indices) :

$$
\sigma(A)=\frac{1}{2} A_{\alpha \beta}\left(a_{\alpha}^{+} a_{\beta}-a_{\beta} a_{\alpha}^{+}\right) .
$$

Thus, $L$ can be specified by some abstract Lie algebra $\lambda$ ( $\lambda$ is a direct sum of Lie algebras of the type of Cartan's list ${ }^{7}$ in their compact real form) and a special faithful unitary representation $\varrho$ of $\lambda$ on an $N$-dimensional complex space (the single particle space) such that

$$
L=\sigma \varrho(\lambda) .
$$

Consequently, any possible $L$ (of our restricted type) can be constructed by a suitable choice of $\lambda$ and $\varrho$, by defining the single particle space (generating the Fock space) by the carrier space of the representation $\varrho$ and by defining the scalar product in that space so that $\varrho$ in unitary.

As a next step we shall make use of the fact that the angular momentum operators are contained in $L$ (alternative ii). This will yield in our construction the angular momentum structure of the single particle space and a corresponding inner structure of $L$.

In general, the angular momentum representation $d$ on the Fock space is given by the single particle representation $\varrho_{\mathrm{a}}\left(\varrho_{\mathrm{a}}\right.$ yields, by decomposition into irreducible parts, the classification $k j \mathrm{~m}$ ) and $\sigma$ through

$$
d=\sigma \varrho_{\mathrm{a}} .
$$

By construction, $\sigma$ and $\varrho$ are faithful representations. In addition, we will assume that also $\varrho_{\mathrm{a}}$ is a faithful representation of so(3), i. e. that we have a nontrivial angular momentum structure in the single particle space. From the relation $d[$ so(3)]C $L$ (alternative ii) ) follows then that

$$
\varrho_{\mathrm{a}}=\left.\varrho\right|_{\lambda^{\prime}} \quad \text { where } \lambda^{\prime}=\varrho^{-1} \varrho_{\mathrm{a}}[\text { so (3) ], }
$$

i. e. $\varrho_{a}$ is the restriction of $\varrho$ to a suitable subalgebra $\lambda^{\prime}$ of $\lambda$ which is of the type so(3), the Lie algebra of rotations.

For the purpose of construction, we may now choose $\lambda^{\prime} C \lambda$ and define $\varrho_{a}$ through Eq. (16), i. e. the angular momentum decomposition of the single particle space will be defined through the analysis of $\varrho$ with respect to the subalgebra $\lambda^{\prime}$. A convenient characterization of the choice of $\lambda^{\prime}$ is obtained with the help of a special faithful representation of $\lambda$ for which we take the fundamental representation $\varrho_{\mathrm{f}}{ }^{19,29}$. (This is the irreducible representation by $n \times n$ matrices in the case of so $(n), \operatorname{su}(n)$ and $\operatorname{sp}(n / 2)$ which define these Lie algebras.) The type of imbedding of $\lambda^{\prime}$ into $\lambda$ is then given by a special $n \times n$ representation of so (3), called $\varrho^{\prime}$.

Summarizing and specifiing more exactly, we define a class of subalgebras $L$ of $L_{N}$ containing the angular momentum operators by the following prescriptions:

1) Choose any semisimple Lie algebra $\lambda$ in its compact real form (abstract type of $L$ ).

2) If the carrier space of the (faithful) fundamental representation $\varrho_{\mathrm{f}}$ of $\lambda$ has dimension $\mathrm{n}$, choose any faithful representation $\varrho^{\prime}$ of so(3) on $C^{n}$ such that
a) $\varrho^{\prime}(e) \in O_{f}(\lambda)$
for $\quad e \in \operatorname{so}(3)$

[i. e. the matrices $\varrho^{\prime}(e)$ must be, e.g., real or symplectic, if $\lambda$ is, e.g., so $(n)$ or $\operatorname{sp}(n / 2)]$;

b) the decomposition of $\varrho^{\prime}$ into irreducible parts

$$
\varrho^{\prime}=d^{l_{1}} \oplus \ldots \oplus d^{l_{r}}
$$

has the property that all $l_{k}(k=1, \ldots, r)$ are either simultaneously integer or simultaneously halfinteger.

[The reason for b) will become clear in 5.] This defines $\lambda^{\prime}=\varrho_{\mathrm{f}}^{-1} \varrho^{\prime}[$ so(3)] as subalgebra of $\lambda$. 
Having $\lambda^{\prime}, \varrho^{\prime}$ is, in turn, given through the restriction of $\varrho_{\mathrm{f}}$ to $\lambda^{\prime}$ :

$$
\varrho^{\prime}=\left.\varrho_{\mathrm{f}}\right|_{\lambda^{\prime}} .
$$

3) Choose any faithful representation $\varrho$ of $\lambda$ on an $N$-dimensional complex space, called $S$.

4) Define the physical single particle space by $S$ and choose the scalar product in $S$ such that $\varrho$ ist unitary.

5) Define the angular momentum structure of $S$, i. e. $Q_{a}$, by that representation of so $(3)$ which is given by the restriction of $\varrho$ to $\lambda^{\prime}[\cong \mathrm{so}(3)]$. The decomposition of $\varrho_{\mathrm{a}}$ into irreducible parts

$$
Q_{\mathrm{a}}=\left.Q\right|_{\lambda^{\prime}}=d^{j_{1}} \oplus \ldots \oplus d^{j_{s}}
$$

defines the angular momentum quantum numbers (2) of the single particle states. The condition 2)b) guarantees that the $j_{k}$ will be always either simultaneously integer or halfinteger allowing the physical interpretation of $\varrho_{a}$ and $d=\sigma \varrho_{\mathrm{a}}$ to be either orbital or total angular momentum.

\section{Structures and Examples of Subalgebras of Type ii)}

For clarification and concreteness, we will now present the subalgebras of $L_{N}$ containing the angular momentum in some more detail, we will give some additional structure and a number of examples.

Let us assume that $\lambda$ has dimension $q$ and that the compact real form of $\lambda$ has a basis $\lambda_{1} \ldots \lambda_{q}$ so that $\lambda_{1} \ldots \lambda_{3}$ constitute $\lambda^{\prime} \cong$ so $(3)$ in the canonical way (i. e. that we have that $\left[\lambda_{k}, \lambda_{l}\right]=\varepsilon_{k l m} \lambda_{m}$, $k, l, m=1, \ldots, 3)$. We defiine further $\varrho\left(\lambda_{\mu}\right)=X^{\mu}$ $\mu=1, \ldots, q)$, where $X^{\mu}$ are antihermitean complex $N \times N$ matrices.

A basis of $L$ is then provided by $L_{1} \ldots L_{q}$ with

$$
L_{\mu}=\sigma \varrho\left(\lambda_{\mu}\right)=\frac{1}{2} X_{\alpha \beta}^{\mu}\left(a_{\alpha}^{+} a_{\beta}-a_{\beta} a_{\alpha}^{+}\right) .
$$

The angular momentum operators corresponding to an infinitesimal rotation around the $k$-axis are given by

$$
L_{k}=X_{\times \beta}^{k} a_{\alpha}^{+} a_{\beta} \quad(k=1,2,3) .
$$

(We used trace $X^{k}=0$.) Thus, the matrices $X_{\alpha \beta}^{k} \quad(k$ $=1,2,3)$ define the representation $\varrho_{\mathrm{a}}$, whose decomposition into irreducible parts [Eq. (18)] yields the single particle angular momentum.

The decomposition of $L$ into spherical tensors [Eq. (5)] amounts to the choice of a special basis of $L$ or $\lambda$. This basis is obtained by considering the adjoint representation $Q_{a d}$ of $\lambda$ (i. e. the representation through the structure constants, see Refs. ${ }^{7}, 19-21$ ), and by performing the analysis of $Q_{\text {ad }}$ with respect to the subalgebra $\lambda^{\prime}$. Calling the restriction of $\varrho_{\text {ad }}$ to $\lambda^{\prime} \varrho_{\text {ad }}^{\prime}$, we write for this analysis

$$
\varrho_{\mathrm{ad}}^{\prime}=\left.\varrho_{\mathrm{ad}}\right|_{\lambda^{\prime}}=d^{J_{1}} \oplus \ldots \oplus d^{J_{p}} .
$$

A determination of the corresponding irreducible subspaces is equivalent to the determination of the basis $B_{v}^{J M}$ [Eq. (5)].

The different types of one and two body operators, as given in Eq. (6), are known through the analysis (21). Any model hamiltonian, generated from $L$, which is hermitean, rotational invariant and contains only one and two body forces is defined by a real linear combination of the hermitean forms of the operators (6) (Hermiticity can be achieved since the $L_{u}$ are antihermitean). These model hamiltonians can be diagonalized by group theoretical methods using the theory of representations of $\lambda$.

The types of decomposition (17), (18) and (20) can be obtained by standard techniques. In a number of cases they are explicitely known from the literature ${ }^{19}$.

In order to give some examples we have quoted in table 1 the structure of subalgebras of $L_{N}$ containing the angular momentum operators for some cases where $\lambda$ is simple and $\varrho$ is irreducible. This structure is characterized through the type of imbedding $\lambda^{\prime}\left(\lambda\right.$ (analysis (17) of $\left.\varrho^{\prime}\right)$, the types of tensor operators constituting $L$ (analysis (21) of $Q_{\mathrm{ad}}^{\prime}$ ) and the dimension $N$ and angular momentum structure of the single particle space $S$ [analysis (18) of $\varrho_{\mathrm{a}}$ ] which must be assumed in order to realize $L=\sigma \varrho(\lambda)$. The representation $\varrho$ is characterized through the maximum weights. (Note that the decompositions (17) and (21) are independent from $\varrho$.) The structure of the decompositions (17), (18) and (21) is always given by the corresponding set of angular momentum values. For su(3), sp(2) and su(4), all possibilities for $\varrho^{\prime}$ are considered, for the other choices of $\lambda$ only the irreducible $\varrho^{\prime}$ is taken into account.

We want to draw the attention to the following interesting examples.

First we recover Elliott's su(3) model by setting $\lambda=\operatorname{su}(3)$ and $\varrho=(L, 0)$. For this choice of $\lambda$ and $\varrho$, the single particle angular momentum structure cor- 
Table 1. Examples of subalgebras $L$ of $L_{N}$ containing the angular momentum operators. The characterization of the subalgebras $L$ is given through the type $\lambda$, the representation $Q^{\prime}$ [analysis of Eq. (17)] defining the imbedding $\lambda^{\prime} \subset \lambda$, the analysis of $\varrho^{\prime}$ ad $=\varrho_{\text {ad }}{\varkappa^{\prime}}^{\prime}[$ Eq. $((21)]$ yielding the decomposition of $L$ into spherical tensors and the representation $\varrho$ defining $L[L=\sigma \varrho(\lambda)]$ for which we quote the maximum weights, the dimension $N$ of the representation space $S$ and the analysis (18) of $\varrho_{a}=\left.\varrho\right|_{\lambda^{\prime}}$ defining the set of shells which have to be assumed in order to constitute $L$. All decompositions are characterized through the possible angular momentum values and their degeneracy (upper index, see Ref. ${ }^{19}$ ).

\begin{tabular}{|c|c|c|c|}
\hline$\lambda=\operatorname{su}(3)$ & $\varrho^{\prime}=(1)$ & \multicolumn{2}{|c|}{$\varrho^{\prime} \mathrm{ad}=(1,2)$} \\
\hline $\begin{array}{l}\varrho \\
(10) \\
(20) \\
(L 0) \\
(11) \\
(21)\end{array}$ & $\begin{array}{l}N=\operatorname{dim} S \\
3 \\
8 \\
\frac{1}{2}(L+1)(L+2) \\
8 \\
15\end{array}$ & $\begin{array}{l}Q_{\mathrm{a}}= \\
1 \\
2,0 \\
L, L- \\
2,1 \\
3,2\end{array}$ &, 1 or 0 \\
\hline$\lambda=\operatorname{sp}(2)$ & $\begin{array}{l}\varrho^{\prime}=(3 / 2) \\
\varrho^{\prime} \text { ad }=(3,1)\end{array}$ & & $\begin{array}{l}\varrho^{\prime}=\left(1 / 2^{2}\right) \\
\varrho^{\prime} \text { ad }=\left(3^{3}, 0\right)\end{array}$ \\
\hline $\begin{array}{lr}\varrho & N \\
(10) & 4 \\
(01) & 5 \\
(20) & 10 \\
(11) & 16 \\
(02) & 14 \\
(30) & 20 \\
(21) & 35 \\
(12) & 40 \\
(03) & 30\end{array}$ & $\begin{array}{l}\varrho_{\mathrm{a}} \\
3 / 2 \\
2 \\
3,1 \\
7 / 2,5 / 2,1 / 2 \\
4,2 \\
9 / 2,5 / 2,3 / 2 \\
5,4,3,2,1 \\
11 / 2,9 / 2,7 / 2 \\
6,4,3,0\end{array}$ & $2,3 / 2$ & $\begin{array}{l}\varrho_{\mathrm{a}} \\
1 / 2^{2} \\
1,0^{2} \\
1^{3}, 0 \\
3 / 2^{2}, 1 / 2^{4} \\
2,1^{3}, 0^{3} \\
3 / 2^{4}, 1 / 2^{2} \\
2^{3}, 1^{9}, 0^{2} \\
5 / 2,3 / 2^{4}, 1 / 2^{6} \\
3,2^{2}, 1^{3}, 0^{2}\end{array}$ \\
\hline
\end{tabular}

\begin{tabular}{|c|c|c|c|c|}
\hline \multicolumn{2}{|c|}{$\lambda=\operatorname{su}(4)$} & $\begin{array}{l}\varrho^{\prime}=(3 / 2) \\
\varrho^{\prime} \text { ad }=(3,2,1)\end{array}$ & $\begin{array}{l}\varrho^{\prime}=\left(1 / 2^{2}\right) \\
\varrho^{\prime} \text { ad }=(3,2,1)\end{array}$ & $\begin{array}{l}Q^{\prime}=(1,0) \\
\varrho^{\prime} \text { ad }=\left(2,1^{3}, 0\right)\end{array}$ \\
\hline$\varrho$ & $N$ & $\varrho_{\mathrm{a}}$ & $\varrho_{\mathrm{a}}$ & $\varrho_{\mathrm{a}}$ \\
\hline$(100)$ & 4 & $3 / 2$ & $1 / 2^{2}$ & 1,0 \\
\hline$(010)$ & 6 & 2,0 & $1,0^{3}$ & $1^{2}$ \\
\hline$(200)$ & 10 & 3,1 & $1^{3}, 0$ & $2,1,0^{2}$ \\
\hline (110) & 20 & $7 / 2,5 / 2,3 / 2,1 / 2$ & $3 / 2^{2}, 1 / 2^{6}$ & $2^{2}, 1^{3}, 0$ \\
\hline (101) & 15 & $3,2,1$ & $1^{4}, 0^{3}$ & $2,1^{3}, 0$ \\
\hline$(020)$ & 20 & $4,2^{2}, 0$ & $2,1^{3}, 0^{6}$ & $2^{3}, 1,0^{2}$ \\
\hline$(300)$ & 20 & & $3 / 2^{4}, 1 / 2^{2}$ & $3,2,1^{2}, 0^{2}$ \\
\hline$(210)$ & 45 & $5,4,3^{2}, 2,1^{2}$ & $2^{3}, 1^{9}, 0^{3}$ & $3^{2}, 2^{3}, 1^{5}, 0$ \\
\hline (201) & 36 & $\begin{array}{l}9 / 2,7 / 2,5 / 2^{2}, \\
3 / 2,1 / 2\end{array}$ & $3 / 2^{6}, 1 / 2^{6}$ & $3,2^{3}, 1^{4}, 0^{2}$ \\
\hline
\end{tabular}

\begin{tabular}{lll}
$\lambda=\operatorname{sp}(3)$ & $\varrho^{\prime}=(5 / 2)$ & $\varrho^{\prime} \mathrm{ad}=(5,3,1)$ \\
\hline$\varrho$ & $\operatorname{dim} S$ & $\varrho_{\mathrm{a}}$ \\
$(100)$ & 6 & $5 / 2$ \\
$(010)$ & 14 & 4,2 \\
$(001)$ & 14 & $9 / 2,3 / 2$ \\
$(200)$ & 21 & $5,3,1$ \\
$(110)$ & 64 & $13 / 2,11 / 2,9 / 2,7 / 2^{2}, 5 / 2,3 / 2,1 / 2$ \\
\hline
\end{tabular}

\begin{tabular}{lll}
\hline$\lambda=\operatorname{so}(7)$ & $\varrho^{\prime}=(3)$ & $\varrho^{\prime} \mathrm{ad}=(5,3,1)$ \\
\hline$\varrho$ & $\operatorname{dim} S$ & $\varrho_{\mathrm{a}}$ \\
$(100)$ & 7 & 3 \\
$(200)$ & 27 & $6,4,2$ \\
$(010)$ & 21 & $5,3,1$ \\
$(002)$ & 35 & $6,4,3,2,0$ \\
\hline
\end{tabular}

noch Tab. 1.

\begin{tabular}{lll}
\hline$\lambda=\operatorname{su}(5)$ & $\varrho^{\prime}=(2)$ & $\varrho^{\prime}{ }^{\prime}=(4,3,2,1)$ \\
\hline$\varrho$ & $\operatorname{dim} S$ & $\varrho_{\mathrm{a}}$ \\
$(1000)$ & 5 & 2 \\
$(2000)$ & 15 & $4,2,0$ \\
$(0100)$ & 10 & 3,1 \\
$(3000)$ & 35 & $6,4,3,2,0$ \\
$(1001)$ & 24 & $4,3,2,1$ \\
\hline$\lambda=\operatorname{sp}(4)$ & $\varrho^{\prime}=(7 / 2)$ & $\varrho^{\prime} \mathrm{ad}=(7,5,3,1)$ \\
\hline$\varrho$ & $\operatorname{dim} S$ & $\varrho_{\mathrm{a}}$ \\
$(1000)$ & 8 & 7,2 \\
$(2000)$ & 36 & $6,4,3,1$ \\
$(0100)$ & 27 & $15 / 2,11 / 2,9 / 2,5 / 2,3 / 2$ \\
$(0010)$ & 48 & $8,5,4,2$ \\
$(0001)$ & 42 &
\end{tabular}

\begin{tabular}{|c|c|c|}
\hline$\lambda=\operatorname{su}(6)$ & $\varrho^{\prime}=(5 / 2)$ & $\varrho^{\prime} \mathrm{ad}=(5,4,3,2,1)$ \\
\hline $\begin{array}{l}\varrho \\
(10000) \\
(20000) \\
(01000) \\
(1] 000) \\
(00100) \\
(10001)\end{array}$ & $\begin{array}{l}\operatorname{dim} S \\
6 \\
21 \\
15 \\
70 \\
20 \\
35\end{array}$ & $\begin{array}{l}\varrho_{\mathrm{a}} \\
5 / 2 \\
5,3,1 \\
4,2,0 \\
13 / 2,11 / 2,9 / 2,7 / 2^{2}, 5 / 2^{2}, 3 / 2,1 / 2 \\
9 / 2,5 / 2,3 / 2 \\
5,4,3,2,1\end{array}$ \\
\hline$\lambda=\operatorname{su}(7)$ & $Q^{\prime}=(3)$ & $\varrho_{\mathrm{ad}}^{\prime}=(6,5,4,3,2,1)$ \\
\hline $\begin{array}{l}\varrho \\
(100000) \\
(200000) \\
(010000) \\
(001000) \\
(100001)\end{array}$ & $\begin{array}{l}\operatorname{dim} S \\
7 \\
28 \\
21 \\
35 \\
48\end{array}$ & $\begin{array}{l}\varrho_{\mathrm{a}} \\
3 \\
6,4,2,0 \\
5,3,1 \\
6,4,3,2,0 \\
6,5,4,3,2,1\end{array}$ \\
\hline$\lambda=\operatorname{su}(8)$ & $\varrho^{\prime}=(7 / 2)$ & $\varrho_{\text {ad }}^{\prime}=(7,6,5,4,3,2,1)$ \\
\hline $\begin{array}{l}\varrho \\
(1000000) \\
(2000000) \\
(0100000) \\
(0010000) \\
(1000001)\end{array}$ & $\begin{array}{l}\operatorname{dim} S \\
8 \\
36 \\
28 \\
56 \\
63\end{array}$ & $\begin{array}{l}O_{\mathrm{a}} \\
7 / 2 \\
7,5,3,1 \\
6,4,2,0 \\
15 / 2,11 / 2,9 / 2,7 / 2,5 / 2,3 / 2 \\
7,6,5,4,3,2,1\end{array}$ \\
\hline
\end{tabular}

responds to an oscillator shell and $L$ contains, besides the (orbital) angular momentum operators $L_{k}(k=1,2,3)$, a set of quadrupole operators $Q^{2 M}$ (corresponding to the $J=2$ part in the decomposition of $\varrho_{\text {ad }}^{\prime}$ ) which allow the definition of a quadrupole force

$$
Q=\sum_{M}(-1)^{M} Q^{2 M} Q^{2-M} .
$$

The eigenvalues of $Q$ can be easily determined by considering representations of su(3) and the chain so (3) $\bigcirc$ su (3). The model (22) is Elliott's su(3). model within one oscillator shell ${ }^{2,3}$ which has been successfully applied to light nuclei and which is 
usually constructed with the help of the symmetry properties of the harmonic oscillator problem. This yields $\varrho$ in a special way, however, it is clear from our construction that the radial parts of the single particle states have not to be oscillator functions (this has been noted by HARVEY ${ }^{3}$ ).

All other examples of subalgebras with their corresponding models can be conceived as generalizations of Elliott's model allowing other configuration spaces and - in general - more types of interaction. A most interesting example occurs for $\lambda=$ su (4) and $\varrho^{\prime}=(3 / 2)$. For $\varrho=(010)$, the decomposition (18) yields the angular momentum structure of the S-D shell, whereas choosing $Q=(110)$ one obtains the structure of the P-F shell with spin orbit splitting. Thus in the first case $d$ has to be interpreted as orbital, in the $2^{\text {nd }}$ case as total angular momentum. In both examples $L$ contains, according to the analysis (21) of $\varrho_{\text {ad }}^{\prime}$, a set of quadrupole and octopole operators $\left(Q^{2 . M}\right.$ and $\left.O^{3 M}\right)$, so that, in addition to (22) and the total angular momentum, (6) contains an octopole-octopole interaction. A detailed investigation of this model will be devoted to a future publication ${ }^{30}$.

Since the $L_{\mu}$ conserve the particle number [see Eq. (19)] $\sigma \varrho$ defines a representation of $\lambda$ on any $n$-body space (generated by $a_{\alpha_{1}}^{+} \ldots a_{\alpha_{n}}^{+}|0\rangle$ ). The decomposition of this representation into irreducible

1 P. W. Anderson, Phys. Rev. 112, 1900 [1958] ; J. Högas. Sen u. H. Feldmann, Nucl. Phys. A 141, 949 [1966] ; R. A. Broglia and B. Sørensen, Nucl. Phys. A 110, 241 [1968] ; J. Garcia and D. Schütte, Nucl. Phys. A 121, 535 [1968].

2 J. P. Elliotr, Proc. Roy. Soc. London Ser. 13, 104 [1959].

3 M. Harvey, in "Advances in Nuclear Physics", ed. by Baranger and Vogt, Vol. 1, Plenum Press, New York 1968, p. 67.

${ }^{4}$ H. J. Lipkin, N. Meshrov, and A. J. Glick, Nucl. Phys. 62, 188 [1965]. - J. C. PARIKH and D. J. Rowe, Phys. Rev. 175, 1293 [1968]. - D. SснÜтTe, Proc. Liperi Summer School in Theoretical Physics, 6, 25 [1966]. - H. R. Petry and D. Schütte, Phys. Letters $38 \mathrm{~B}, 167$ [1972].

5 D. Agassi, Nucl. Phys. A 116, 49 [1968]. - D. Schütte und K. Bleuler, Nucl. Phys. A 119, 221 [1968]. - K. Bleuler, A. Friedrich, and D. Schütte, Nucl. Phys. A 126, 628 [1969]. - H. R. Petry and D. Schütte, Nucl. Phys. A 195, 623 [1972].

${ }^{6}$ D. Agassi, Nucl. Phys. A 119, 497 [1968].

7 S. Helgason, Differential Geometry and Symmetric Spaces, Academic Press, New York 1962.

8 G. RacaH, Phys. Rev. 76, 1352 [1949].

9 B. H. Flowers, Proc. Roy. Soc. London A 212, 248 [1952].

10 B. R. Judd, Operator Techniques in Atomic Spectroscopy, McGraw-Hill, New York 1963. parts yields a special basis in the $n$-body space and defines quantum numbers in addition to angular momentum. In Elliott's su(3) model, such basis systems have shown up to be useful for truncation even for the case of realistic forces ${ }^{3}$, and it is clear that one may try such applications also for the generalized subalgebras constructed here.

Besides that, a number of other examples of our table should be useful for more schematic investigations (such as test of approximation techniques) :

E. g. we have the (00100) representation of su (6) and the $(0010000)$ representation of su(8) allowing the construction of models containing four $(J=$ $2,3,4,5)$ resp. six $(J=2,3,4,5,6,7)$ different types of model interactions on Fock spaces which contain a rather rich shell structure.

Finally, it should be mentioned that for a single $j$ shell, we recover the wellknown examples of subalgebras of type ii). They are given by the choices $\lambda=\operatorname{so}(2 j+1)[$ resp. $\operatorname{sp}((2 j+1) / 2)]$ for $j$ integer (resp. $j$ halfinteger), $\varrho^{\prime}=d^{j}$ and $\varrho=\varrho_{\mathrm{f}}$, Refs. ${ }^{15,21}$. Considering direct sums, these subalgebras can be generalized to more shells ${ }^{31}$. For the atomic nucleus, also the choice $\lambda=g_{2}, \varrho^{\prime}=d^{3}, \varrho=\varrho_{\mathrm{f}}$ has found interesting applications ${ }^{15}$.

It is a pleasure to thank Professor K. BLEULER for his interest and for many fruitful discussions concerning this work.

11 B. G. Wybourne, Spectroscopic Properties of the Rare Earths, John Wiley, New York 1965. - B. G. BybournE and P. H. Butler, J. Phys. 30, 181 [1968].

12 S. Feneuille, J. Phys. 28, 61 [1967] ; 28, 315 [1967] ; 28, 497 [1967].

13 M. Moshinsky, Group Theory and the Many Body Problem, Gordon and Breach, New York 1965.

14 P. Kramer and T. H. Seligman, Nucl. Phys. A 123, 161 [1969] ; A 136, 545 [1969] ; A 186, 49 [1972].

15 B. R. Judd, Group Theory in Atomic Spectroscopy, in "Group Theory and its Applications", E. M. Loebl, Academic Press, New York 1968.

16 B. G. Wybourne, Symmetry Principles and Atomic Spectroscopy, John Wiley, New York 1970.

17 P. Kramer and M. Moshinsky, Group Theory of Harmonic Oscillators and Nuclear Structure, in "Group Theory and its Applications", E. M. Loebl, Academic Press, New York 1968.

18 G. RaCAH, Group Theory and Spectroscopy, Erg. Exact. Naturwiss. 37, 28 [1965].

19 M. Hamermesh, Group Theory and its Applications to Physical Problems, Addison-Weseley, Reading, Mass. 1962.

20 R. Hermann, Lie Groups for Physicists, W. A. Benjamin, Inc., New York and Amsterdam 1966.

21 H. J. Lipkin, Lie Groups for Pedestrians, North Holland, Amsterdam 1965. 
22 U. FANO and G. RACAH, Irreducible Tensorial Sets, Academic Press, New York 1959.

23 R. Arvieu, Trieste Lectures 1969, ed. by Int. Atomic Energy Ag., Vienna 1970, p. 499.

24 A. EDmonds, Angular Momentum in Quantum Mechanics, Univ. Press, Princeton, New Jersey 1968.

25 D. SCHÜTTE, Habilitationsschrift, Bonn 1969.

26 K. T. Hecht, Nucl. Phys. 63, 177 [1965].
27 E. P. Wigner, Phys. Rev. 51, 106 [1937].

28 B. Bremond, Nucl. Phys. A 113, 257 [1968].

29 J. Tits, Tabellen zu den einfachen Lie Gruppen und ihre Darstellungen, Lecture Notes in Mathematics No. 40, Springer-Verlag, Berlin 1968.

30 D. SChubert and D. SChütTe, to be published.

31 R. Arvieu and S. A. Moskowski, Phys. Rev. 145, 830 [1966].

\title{
Äquivalenz der $\mathrm{N}^{*}$-Beiträge in der Starken Kopplung $\mathrm{zu}$ einem skalaren $\mathrm{N}-\mathrm{N}$-Potential
}

\author{
Siegfried WAgner * \\ Institut für Theoretische Kernphysik der Universität Bonn \\ (Z. Naturforsch. 28 a, 403-407 [1973] ; eingegangen am 19. Oktober 1972)
}

KonRAD BLeUler zum 60. Geburtstag gewidmet

\begin{abstract}
The coupled-channel contribution of $\mathrm{N}_{33} *$ isobars, which appear in the solutions of the fixedsource strong coupling field equations, are equivalent to an effective scalar $\mathrm{N}-\mathrm{N}$ potential with the range of the $\sigma$-exchange in usual $\mathrm{OBE}$ models.
\end{abstract}

\section{Die Lösungen der starken Kopplung für das NN-System}

Das Verfahren der „starken Kopplung“ wurde 1940 von WeNTZEL ${ }^{1}$ in die Feldtheorie eingeführt und in dem darauffolgenden Jahrzehnt von den Schulen Wentzels und Paulis ${ }^{2}$ sowie von BleuLER $^{3} \mathrm{zu}$ einer umfassenden Theorie der starken Wechselwirkung ausgearbeitet. Sowohl das MesonNukleon- als auch das Nukleon-Nukleon- (Deuteron und Streuung) und das Vielkörperproblem der Kernmaterie führte für verschiedenartige Kopplungsansätze zu geschlossenen Ausdrücken. Später wurden auch die seltsamen Teilchen einbezogen ${ }^{4}$ sowie der gruppentheoretische Aspekt ausgearbeitet ${ }^{5}$.

Eine notwendige Voraussetzung der Theorie ist die Existenz eines Formfaktors für die Nukleonen. Es werden im Gegensatz zur gewöhnlichen Störungsreihe (die für physikalische Kopplungskonstanten bekanntlich divergiert) Entwicklungen nach fallenden Potenzen von $g^{2}$ angesetzt. Da diese Theorie nur für den skalaren Fall in den höheren Ordnungen untersucht worden ist ${ }^{6}$, hat sich der Name „starke Kopplung " für die Lösungen der Mesonenfeldtheorie in der Ordnung $1 / g^{2}$ eingebürgert, worauf auch die „strong coupling groups" fußen. Für das Meson-

* Diese Arbeit wurde finanziert vom Bundesministerium für Wissenschaft und Forschung.

Sonderdruckanforderungen an Dr. S. WAGNER, Institut für

Theoret. Kernphysik der Universität Bonn, D-5300 Bonn,

Nußallee 16.
Nukleon-System erhält man in dieser Näherung ein unendliches System von isobaren Nukleonzuständen mit identischen halbzahligen Werten des Spins und Isospins $j$ mit der Anregungsenergie:

$$
E_{\mathrm{Is}}=\varepsilon \mu\left[j(j+1)-\frac{3}{4}\right] ; \quad\left(j=\frac{1}{2}, \frac{3}{2}, \frac{5}{2}, \ldots\right)
$$

mit $\mu=m_{\pi} / \hbar c$ und

$$
\varepsilon=\frac{3}{4} a \mu / f^{2},
$$

wobei $f$ die im Hamiltonian angesetzte unrenormierte Kopplungskonstante ist, die mit der physikalischen Kopplungskonstante $g$ zusammenhängt:

$$
f^{2}=9\left(m_{\pi} / 2 m_{\mathrm{N}}\right)^{2} g^{2} / 4 \pi
$$

und $a$ der Radius der Quellfunktion ist. Es ist bemerkenswert, daß der Limes verschwindenden Formfaktors in (1) zu dem unsinnigen Ergebnis eines nicht definierten Spins führt, was bei schwacher Kopplung nicht auftritt. Die Quantenzahlen des ersten angeregten Zustandes stimmen mit den ein Jahrzehnt nach Aufstellung dieser Theorie experimentell entdeckten $\mathrm{N}_{3 / 2}^{*}$-Nukleon-Isobaren überein.

Das Auftreten der angeregten Nukleonen in den Lösungen der starken Kopplung für das Zweinukleonenystem führt auf ein Mehrkanalproblem. In niedrigster Ordnung der Störungsreihe der starken Kopplung erhält man:

$$
\begin{gathered}
\sum_{n^{\prime}}\left[-\mathrm{d}^{2} / \mathrm{d} x^{2}+L_{n}\left(L_{n}+1\right) / x^{2}+V_{n n^{\prime}}^{J P T}(x)\right] F_{u^{\prime}}^{T J P}(x, E) \\
=\eta_{n} F_{n}^{J T P}(x, E),
\end{gathered}
$$

